\title{
Observing Structural Dynamics and Measuring Chemical Kinetics in Low Dimensional Materials Using High Speed Imaging
}

\author{
A I Kirkland ${ }^{1,2 *}$, C. S. Allen ${ }^{1,2}$, E. Besley ${ }^{3}$, C. Huang ${ }^{1,2}$, J. Kim $^{1,2}$ S. Skowron ${ }^{3}$ and J. Warner ${ }^{1}$ \\ 1. Department of Materials, Parks Road, Oxford, UK. \\ 2. Electron Physical Sciences Imaging Centre (ePSIC), Diamond Light Source Ltd, Didcot, UK. \\ 3. School of Chemistry, University of Nottingham, University Park, Nottingham, UK. \\ * Corresponding author: angus.kirkland@materials.oc.ac.uk
}

The advent of direct counting electron detectors for use with transmission electron microscopy revolutionized the field of cryo-electron microscopy for structural biology [1]. Their very high quantum detection efficiency and low read-out noise allows very high signal to noise images to be acquired and frame rates in the $\mathrm{kHz}$ range enables compensation of the effects of sample drift. However, in applications for materials science these detectors are yet to have the same level of impact. Efforts to date have concentrated on using direct electron detectors for 4D-STEM experiments in which the full farfield electron diffraction pattern is collected for every probe position in a STEM scan [2-4]. An alternative potential application for direct electron detectors which is only beginning to be explored utilizes their high temporal resolution. In this work we will describe imaging of dynamics events in low dimensional materials at low accelerating voltages with ms exposure times. We will further show how the large datasets generated can be analysed using machine learning to yield information about the stability of individual defect structures that can be directly compared to those predicted from quantum chemical calculations of reaction pathways.

Figure 1 shows a typical short time series of images of a complex defect in monolayer graphene that undergoes a series of structural changes together with the labelled outputs from a suitably trained neural network. All images were recorded at $80 \mathrm{kV}$ using a Medipix 3 direct electron sensor operating counting mode with a maximum readout rate of $1.2 \mathrm{kHz}$ [5]. The defect transitions shown are the interconversion between the $\mathrm{V}_{2}$ (555-777) defect consisting of a symmetric circular arrangement of 5 and 7 member rings and the $\mathrm{V}_{2}$ (5555-6-7777) with two orthogonal mirror axes. This particular rearrangement is mediated by a Stone Wales bond rotation with a predicted energy barrier between the two topologies of $17 \mathrm{eV}$ for both forward and reverse transformations consistent with their equivalent experimental lifetimes. Moreover both defects have as their origin an unstable divacancy formed by the elimination of two $\mathrm{C}$ atoms following electron irradiation.

We will discuss these and other transformations in graphene in detail and show how analysis of suitably large datasets (for statistical significance), varying electron flux and sample temperature independently can provide an insight into chemical kinetics at the nanoscale. Finally recent work exploring the dynamics of adatoms at graphene edges will be presented [6]. 


\section{References:}

[1] E Callaway, Nature 525 (2015), p. 172.

[2] J Song et al., Scientific Reports, in press, (2019).

[3] Y Jiang et al., Nature 559 (2018), p. 343.

[4] H Yang et al., Ultramicroscopy 180 (2017), p. 173.

[5] JA Mir et al., Ultramicroscopy 182 (2017), p. 44.

[6] The authors acknowledge Diamond Light Source for access and support in use of the electron Physical Science Imaging Centre that contributed to the results presented here.
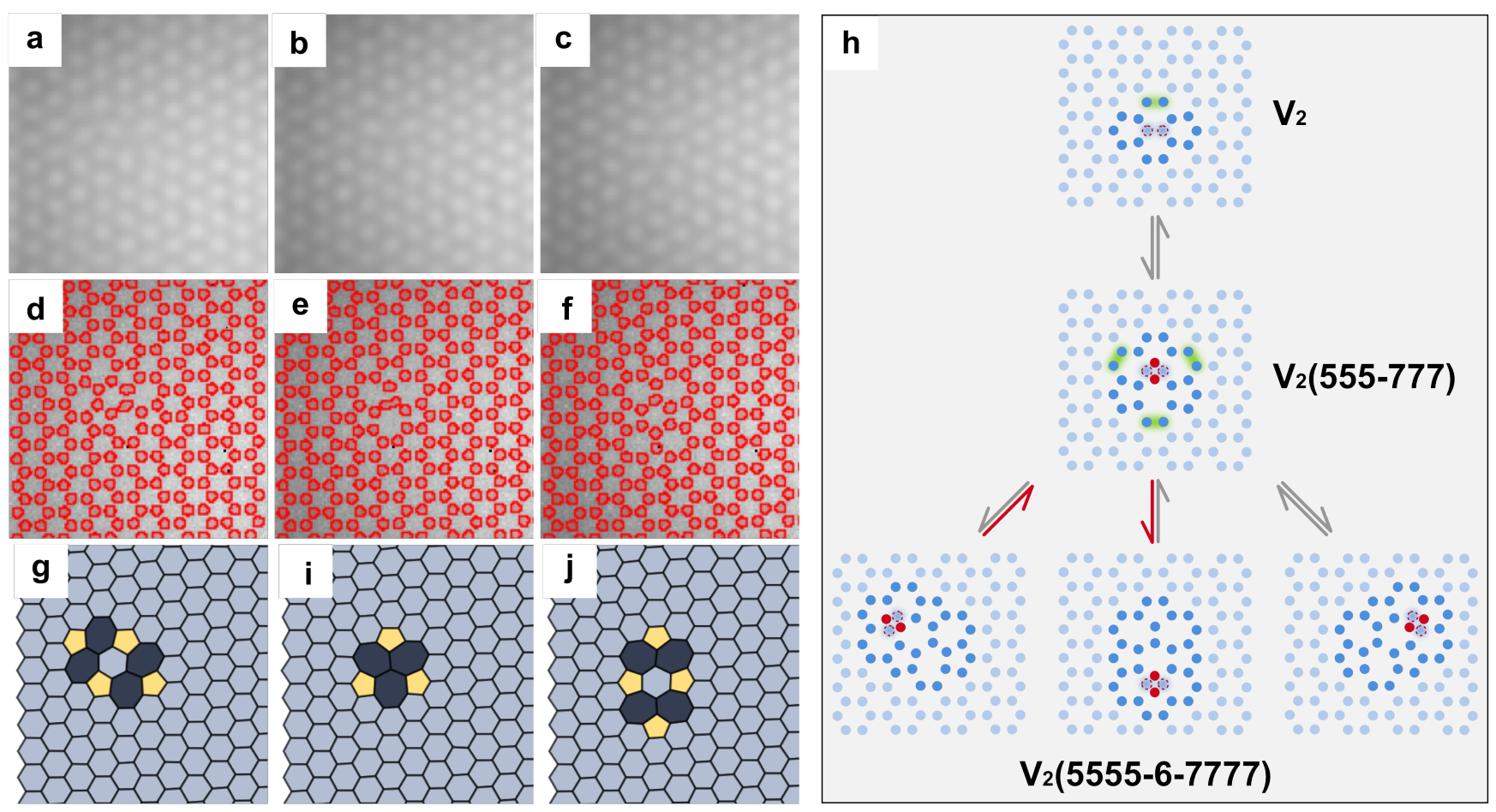

Figure 1. (a-c) High resolution TEM images of monolayer graphene recorded at $80 \mathrm{kV}$ with using an average of $5 \times 1 \mathrm{~ms}$ exposures recorded on a Medipix 3 sensor (d-f) Output from a trained neural network using an output label locating coordinates in a graphene lattice. (g,i,j) Automated ring topology detection with 5, 6 and 7-membered rings displayed as polygons of different colours. (h) Calculations showing the interconversion pathways between the defects types observed. Stone Wales bond rotations are highlighted in red. 